\title{
Visual consequences of molecular changes in the guanylate cyclase activating protein
}

\author{
Andrew Stockman ${ }^{1}$, G. Bruce Henning ${ }^{1}$, Anthony T. Moore ${ }^{1,2}$, Andrew R. Webster ${ }^{1,2}$, Michel \\ Michaelides $^{1,2}$ and Caterina Ripamonti ${ }^{1}$ \\ ${ }^{1}$ UCL Institute of Ophthalmology, University College London, 11-43 Bath Street, London EC1V \\ 9EL, England \\ ${ }^{2}$ Moorfields Eye Hospital, City Road, London, EC1V 2PD, England \\ Address for correspondence: UCL Institute of Ophthalmology, University College London, 11-43 Bath \\ Street, London EC1V 9EL, England
}

\begin{abstract}
Purpose:

In this study, we characterize and model changes in visual performance associated with a Tyr99Cys substitution in the guanylate cyclase activating protein (GCAP1) in four family members aged between 39 and 55 years. Guanylate cyclase and its activating protein are molecules in the visual transduction pathway that restore cyclic GMP (cGMP) following its light-activated hydrolysis. The mutation causes an excess of cGMP in the dark and results in progressive photoreceptor loss.
\end{abstract}

\section{Methods:}

L-cone temporal acuity was measured as a function of target irradiance; and L-cone temporal contrast-sensitivity was measured as a function of temporal frequency.

\section{Results:}

All four GCAP1-mutant family members show sensitivity or acuity losses relative to normal observers. The data for the youngest family member are consistent with an abnormal speeding up of the visual response relative to normals, but those for the older members show a progressive higherfrequency sensitivity loss consistent with a slowing down of their response.

\section{Conclusions:}

The speeding up of the visual response in the youngest observer is consistent with the Tyr99Cysmutation resulting in the more rapid replacement of cGMP after light exposure, and thus in a reduction of temporal integration and relative improvement in high frequency sensitivity compared to normals. The high-frequency losses in the older observers are consistent with their vision being further limited by the interposition of some sluggish process. This might result from some residual or malfunctioning molecular process limiting transduction within damaged photoreceptors, or from an active or passive postreceptoral reorganization caused by the paucity of functioning photoreceptors.

Keywords: Guanylate cyclase, RetGC1, Guanylate cyclase activating protein, GCAP1, flicker sensitivity, critical flicker fusion, temporal processing. 


\section{INTRODUCTION}

Estimating the speeds of molecular processes from the temporal sensitivity differences between normal observers and observers with genetically-characterized molecular defects is a powerful way of quantitatively linking molecular processes to visual performance. Here, we investigate defects in the guanylate cyclase activating protein.

The first and arguably most important step in human vision is the transduction of a photon into an electrical signal, which is achieved within the photoreceptor by a cascade of molecular processes initiated by the absorption of a photon by the chromophore, 11-cis-retinal. A crucial step in this cascade is the activation of the effector molecule-the phosphodiesterase enzyme (PDE6), which reduces the cytoplasmic concentration of cyclic GMP (cGMP) by catalyzing its hydrolysis into GMP. The reduction in CGMP leads to the closure of the cyclic-nucleotide-gated (CNG) channels in the plasma membrane, so blocking the inward flow of $\mathrm{Na}^{+}$and $\mathrm{Ca}^{2+}$ ions and thereby initiating membrane hyperpolarization and the neural response. This study is concerned with the guanylate cyclase activating protein (GCAP) that stimulates guanylate cyclase (RetGC) to resynthesize cGMP following its light-activated hydrolysis by the activated phosphodiesterase (PDE6*), and thus helps to shape the photoresponse. Details of the transduction cascade can be found in several reviews. ${ }^{1-5}$ Since the activity of GCAP and RetGC is $\mathrm{Ca}^{2+}$ sensitive, and $\mathrm{Ca}^{2+}$ concentration decreases in the light, these molecules together contribute to sensitivity regulation through a negative feedback loop that opposes the effect of photon absorption on cGMP levels. ${ }^{6,7}$

There are two types of membrane-bound guanylate cyclases expressed in rod and cone photoreceptors: RetGC1 encoded by GUCY2D, and RetGC2 encoded by GUCY2F., ${ }^{8,9}$ RetGC1 and RetGC2 are themselves regulated by $\mathrm{Ca}^{2+}$-binding GCAP proteins, of which there are three isoforms: GCAP1 encoded by GUCA1A, GCAP2 by GUCA1B and GCAP3 by GUCA1C. ${ }^{10-12}$ Our study involves four observers with the Tyr99Cys mutation in GCAP1. ${ }^{13}$

\section{GCAP1 and the Tyr99Cys mutation}

GCAP1 is found mainly in cones but also in rod photoreceptors, in both of which it stimulates RetGC1 to resynthesize cGMP when light reduces the intracellular $\mathrm{Ca}^{2+}$ levels. ${ }^{11,14,15}$ GCAP proteins have four "EF" hands made up of a helix-loop-helix conformation: EF1 is modified to interact with RetGCs, ${ }^{16}$ while EF2, EF3, and EF4 bind with $\mathrm{Ca}^{2+}$ (see Figure 8 of Hunt, Buch \& Michaelides ${ }^{17}$ ). The Tyr99Cys mutation disrupts the EF3 binding arm causing the mutant GCAP1 to continue stimulating RetGC1 even in the dark when $\mathrm{Ca}^{2+}$ levels are high and even in the presence of calcium-loaded wildtype (normal) GCAPs. ${ }^{18,19}$. 
Clinically, the Tyr99Cys mutation of GCAP1 leads to dominant cone-rod dystrophy, and less frequently cone dystrophy and isolated macular cone loss. ${ }^{13}$ The initial symptoms are mild photophobia, reduced central vision and visual acuity, and a loss of colour vision that usually becomes apparent between the ages of 20 and 40 years. Electrophysiologically there is ultimately a reduction in the amplitude of photopic ERGs to $30-\mathrm{Hz}$ flicker and single flash, but without increases in implicit time (the latencies to peak responses); and scotopic ERGs may be reduced if there is rod involvement. Visual acuity gradually deteriorates with age, but peripheral vision may be relatively preserved in patients with a cone dystrophy or macular dystrophy phenotype..$^{13,20-22}$

\section{GCAP, CGMP and the visual response: in vivo murine models}

The Tyr99Cys-mutant GCAP1 constitutively activates RetGC1 even when the $\mathrm{Ca}^{2+}$ concentration is high and so produces higher than normal concentrations of cGMP at lower light levels. Because of the enhanced activity at these levels, we might also expect a more rapid than normal replacement of cGMP following light activation. In addition, the excess of cGMP in the dark should increase the $\mathrm{Ca}^{2+}$ level, which might enhance the size of dim flash responses.

Much of the relevant evidence concerning the effects of GCAP comes from rod measurements made in vivo in transgenic Tyr99Cys-mutant GCAP1 mice (which also have wild-type GCAP1), and in knockout mice lacking GCAP1 and GCAP2. The key types of rod data collected in mice are typically rod suction-electrode current recordings for (a) low-intensity, single flashes that produce singlephoton responses and (b) series of single flashes from low to saturating intensity levels. Two key variables extracted from these measurements are: (a) $\tau_{\text {rec }}$ (or $\tau_{\text {dim }}$ ), the time constant of exponential recovery after the peak single-photon response, and $(b) \tau_{D}$, (or $\tau_{\text {sat }}$ ) the dominant time constant of exponential recovery after the rod responses to bright flashes reach saturation. In transgenic Tyr99Cys-mutant GCAP1 mice, ${ }^{23}$ the single-photon response is about twice the size of the normal response, yet the exponential rates of decay after the peak are similar in the two cases (analysing the data from Figure $6 \mathrm{~b}$ of Olshevskaya et al., ${ }^{23}$ we estimate time constants, $\tau_{\text {rec, }}$ of about 250 and $230 \mathrm{~ms}$ for Tyr99Cys-mutant and normal responses, respectively). By comparison, the amplitude of the single-photon response in mice lacking both GCAP1 and GCAP2 is about 5 times greater than normal and the decay after the peak slower $\left(\tau_{\text {rec }}=313 \mathrm{~ms}\right){ }^{7,24}$ Clearly, GCAP1 and Ca ${ }^{2+}$ feedback to RetGC is important in shortening the offset of the dark adapted rod flash response, ${ }^{24,25}$ but the Tyr99Cys-mutation seems to have relatively little effect on the speed of the offset compared to the wild-type (normal) rod response. 
In contrast, the dominant rate of recovery following rod response saturation is the same in mice lacking GCAP1/GCAP2 and in normal wild-type mice $\left(\tau_{D}=240 \mathrm{~ms}\right)$, which suggests that at least in the recovery from saturation $\mathrm{Ca}^{2+}$-dependent cGMP resynthesis is not rate limiting., ${ }^{75-27}$ Similarly, the Tyr99Cys-mutatation seems to cause only a modest increase in the duration of saturating response (see Figure 6a of Olshevskaya et al., ${ }^{23}$ ).

On the basis of these murine rod measurements, we might expect the Tyr99Cys-mutant GCAP1 to have relatively little effect on our temporal sensitivity measurements. However, the psychophysical data are consistent with a speeding up of the photopic visual response in our youngest Tyr99Cys-mutant observer.

\section{Light adaptation and temporal sensitivity}

Light adaptation or sensitivity regulation, which is one of the most important functions of the cone photoreceptor, enables it to respond to small proportional changes in intensity over the enormous range of light levels to which it can be exposed in the environment. Adaptation is achieved in large part by a speeding up of the visual response as the light level increases, and thus a shortening of the integration time (see Equation [3], below, and for discussion see Stockman et $a l .^{28}$ ). The murine data apart (see above), one of the molecular mechanisms responsible for this change in normal vision is probably the increased activity of RetGC and GCAP in the light as the $\mathrm{Ca}^{2+}$ level falls, which leads to the restoration of cGMP (see Table 1 of Pugh Nikonov \& Lamb ${ }^{2}$ ). The effects of speeding up the photoreceptor response can be clearly observed in psychophysical measures of temporal sensitivity (i.e. as changes in an observer's sensitivity to flickering lights)most characteristically as relative increases in high-frequency sensitivity with increasing mean light level..$^{29-31}$

If we are correct in supposing that the increased $\mathrm{Ca}^{2+}$-dependent activity of RetGC and GCAP measurably speeds up the cone visual response, then observers with the Tyr99Cys-mutant GCAP1, which leads to a more rapid replacement of cGMP at lower light levels, might be expected to be relatively better at detecting higher temporal frequencies than normal observers. A clear complication in linking the molecular change to visual performance is that this type of cone-rod dystrophy is progressive. ${ }^{21,22}$ Thus, although the initial direct visual effects of the molecular defect and the excess production of cGMP might be apparent in the visual behaviour of younger observers, in older observers any effects are likely to be hidden by increasing loss or damage. Another important question, then, is what form does the increasing loss or damage take? Is it consistent 
simply with loss of photoreceptors? Or with a change in the function of the remaining photoreceptors or postreceptoral pathways? Or, indeed, all three?

Our GCAP1-mutant observers are limited to a single generation within the same family, as a result of which the age range is limited to $39-55$ years. Nevertheless, we find a pattern of loss in the family of observers with the Tyr99Cys-mutant GCAP1 that is consistent with the imposition of an increasingly sluggish low-pass filter (in other words, a process that increasingly attenuates or blocks higher temporal-frequency flicker relative to low-frequency flicker). We did not have access to the younger generation of the family, who have not as yet been diagnosed.

\section{METHODS}

\section{Observers}

The experimental group consisted of four observers in the same family with a Tyr99Cys substitution in GCAP1 ${ }^{21}$. The observers' gender, age at testing, genotype and visual acuities in the tested eye are given in Table 1. Adults with normal or corrected to normal visual acuity and normal colour vision provided representative control data. (The normal observers all had normal colour vision as assessed by the Farnsworth-Munsell 100 hue test and other standard colour vision tests.)

\section{TABLE 1}

\begin{tabular}{ccccc}
\hline Observer & Sex & Age & Genotype & Visual Acuity \\
\hline GP1 & M & 39 & GUCA1A p.Tyr99Cys & $6 / 12$ \\
GP2 & F & 41 & GUCA1A p.Tyr99Cys & $6 / 12$ \\
GP3 & M & 51 & GUCA1A p.Tyr99Cys & $6 / 9$ \\
GP4 & F & 55 & GUCA1A p.Tyr99Cys & $6 / 12$ \\
\hline
\end{tabular}

The colour-vision test results for all the affected observers were broadly consistent with a generalised loss of colour vision. GP1 and GP2 had low discrimination on the FM-100 hue test, with deficits along tritan and protan lines (S-cone and L-cone deficiencies, respectively), while GP4 had very low discrimination (GP3 did not take this test). Red-green Rayleigh anomaloscope settings were highly variable, and were sometimes consistent with normal or protanomalous settings (GP2); 
sometimes with deuteranomalous or protanomalous (abnormal M-cone and L-cone, respectively) settings (GP1 and GP3) and sometimes they were relatively normal (GP4).

All the studies conformed to the standards set by the Declaration of Helsinki, and the procedures have been approved by local ethics committees at Moorfields Eye Hospital and at University College London.

\section{Apparatus}

The optical system for the experiments has been described in detail elsewhere ${ }^{32}$. Briefly, the optics comprised two channels of a standard Maxwellian-view system with a 2-mm exit pupil illuminated by a $900-\mathrm{W}$ Xe arc lamp. One channel was used to produce a circular background field of $9^{\circ}$ diameter, and the second, to produce a concentric 'target' field with a diameter of $4^{\circ}$. The wavelengths of the target and background were determined by interference filters (Ealing, or Oriel) with full bandwidth at half-maximum transmission of between 7 and $11 \mathrm{~nm}$ inserted into collimated beams in each channel. The radiance in each channel was determined by a combination of neutraldensity filters (Oriel) also inserted into collimated beams, and by the rotation, under computer control, of a circular, variable-neutral-density filter (Rolyn Optics, Covina, CA) located near a focus within the target channel.

Sinusoidal variation in the target radiance was produced by pulse-width modulation of the target beam by a fast, liquid-crystal, light shutter located in the target beam and running at $400 \mathrm{~Hz}$ with rise and fall times faster than $50 \mu$ s (Displaytech, Longmont, $\mathrm{CO}$ ) thus producing effectively rectangular pulses of variable width at a fixed frequency of $400 \mathrm{~Hz}$. The pulse width was varied sinusoidally under computer control using programmable timers (Data Translation, DT2819) to produce sinusoidal stimuli at the desired visible frequencies and at signal modulations up to $92 \%$. (Frequencies near the $400-\mathrm{Hz}$ rectangular-pulse frequency and above were much too high to be resolved, so that observers saw only the sinusoidally-varying stimuli produced by the variation of the pulse width.)

The patient's head was fixed to the system by a hardened dental impression mounted on a milling-machine head adjusted to locate the exit pupil of the optics in the centre of, and in the plane of pupil of, the patient's right eye. 


\section{Stimuli}

We were primarily interested in L-cone responses and measured their temporal properties by sinusoidally flickering the $4^{\circ}$ target. We refer to the amplitude of the flicker relative to the mean radiance as the "modulation", $m$, which is defined as the conventional Michelson contrast:

$$
m=\frac{I_{\max }-I_{\min }}{I_{\max }+I_{\min }}
$$

where $I_{\max }$ and $I_{\min }$ are the maximum and minimum radiances of the stimulus, respectively. Thus, for sinusoidal flicker, the flickering waveform, $A(t)$, is given by:

$$
A(t)=\bar{R}\{1+m \sin (2 \pi f t)\},
$$

where $\bar{R}$ is the mean radiance and $f(\mathrm{~Hz})$ is the rate of flicker. The modulation, $m$, could be varied under computer control, and was limited to a maximum of $92 \%$.

L-cone stimuli. A flickering target of $4^{\circ}$ of visual angle in diameter and $650-\mathrm{nm}$ wavelength was presented in the centre of a $9^{\circ}$ diameter background field of $481 \mathrm{~nm}$. Fixation was central. The 481$\mathrm{nm}$ background, which delivered $8.24 \log$ quanta s $\mathrm{s}^{-1} \mathrm{deg}^{-2}$ at the cornea (1.37 $\log _{10}$ photopic trolands or $2.53 \log _{10}$ scotopic trolands), served mainly to suppress the rods, but also selectively desensitized the $\mathrm{M}$-cones relative to L-cones at lower target radiances. The background was present for all the experiments reported here. For the critical fusion frequency (CFF) measurements, the target intensity was varied from 6.5 to $11.5 \log _{10}$ quanta s${ }^{-1} \mathrm{deg}^{-2}$. These conditions isolate the L-cone response over most of the intensity range, but, at high intensities, the $\mathrm{M}$-cones may also contribute to flicker detection but we were not concerned about the possibility of a mixed $\mathrm{M}$ - and L-cone response at higher levels. For the temporal contrast sensitivity measurements, the target radiance was set to time-averaged radiances of either $8.28,9.30$ or $10.28 \log _{10}$ quanta s${ }^{-1} \mathrm{deg}^{-2}$, which we shall refer to as the low, medium and high levels, respectively.

\section{Procedures}

All observers light adapted to the background and target for 3 minutes before any measurements. They interacted with the computer that controls the apparatus by means of an eight-button keypad, and received information and instructions via tones and a computer-controlled voice synthesizer. Each experiment was repeated three times usually on separate days. The mean of the results for each experimental run was averaged and the standard error determined. The visual stimulus, focused in the plane of the pupil, was the only visible light source for the observers in an otherwise dark room. The image of the source in the plane of the observers' pupils was always less 
than the minimal pupil size so that retinal illumination was not affected by pupil size. The method of adjustment was used to measure visual responses in the experiments.

Two types of temporal sensitivity experiments were performed: (1) critical fusion frequency (CFF) was measured as a function of target radiance, and (2) temporal contrast-sensitivity was measured as a function of temporal frequency at the three mean target radiances.

Critical fusion measurements. The target modulation was held fixed at $92 \%$ and the time-average radiance set to values ranging from about 6 to $11 \log _{10}$ quanta $s^{-1} \mathrm{deg}^{-2}$ by the experimenter's inserting neutral-density filters into the target channel. At each target radiance, the observer adjusted the rate of flicker up or down by means of buttons to determine the highest frequency of flicker at which flicker was just visible - their flicker "threshold" or critical fusion frequency (CFF) and indicated that they were satisfied with their adjustment by pushing a third button. The observer then changed the flicker frequency away from their threshold setting and re-determined the highest frequency at which flicker was just visible. The process was repeated three times before the mean radiance of the target was changed. All three settings and their mean were stored in the computer. The experiment was repeated on three separate occasions for the normal observers and, depending on availability, on two or three separate occasions for the affected observers.

Temporal contrast sensitivity measurements. The frequency of the flickering target was fixed at values ranging from 0.5 to $50 \mathrm{~Hz}$. The mean radiance of both the background and target were also fixed. The observers adjusted the modulation of the flickering stimulus ( $m$ in Equation 2 ) to determine the lowest modulation at which a given flicker was just visible. Modulation could be varied up or down in large or small steps depending on the button pressed. Again they indicated that they were satisfied with their adjustment by pushing a third button. The observer then moved the modulation away from their setting and re-determined their modulation "threshold". The process was repeated three times before the flicker frequency of the target was automatically changed by the computer. All three settings and their mean were stored in the computer and the experiment was repeated on three separate occasions. The average and standard error of the means obtained on the three different occasions for the normal observers and, depending on availability, on two or three separate occasions for the affected observers are reported.

\section{Calibration}

The radiant fluxes of the target and background fields were measured at the plane of the exit pupil using an UDT radiometer, calibrated by the manufacturer (Gamma Scientific) against a standard traceable to the US National Bureau of Standards. The neutral-density filters (and circular 
neutral-density wedge) were calibrated in the optical system, separately for each wavelength used, using the radiometer. The target radiances are reported as time-averaged values. Neutral density filters, fixed and variable, were calibrated in situ for all test and field wavelengths used. A spectroradiometer (EG\&G) was used to measure the centre wavelength and the bandwidth at half amplitude of each interference filter in situ.

\section{RESULTS}

\section{L-cone critical flicker fusion}

Figure 1 shows L-cone CFF (temporal acuity) data for the four observers with Tyr99Cys-mutant GCAP1 plotted on the linear ordinate as a function of $\log _{10}$ target radiance. The CFF at each target radiance is the highest frequency at that radiance at which the target appears to flicker. The data for GP1 to GP4, whose ages increase from GP1 to GP4, are indicated by green triangles, purple circles, yellow inverted triangles, and blue diamonds, respectively. The mean L-cone CFF data for 12 observers with normal vision are plotted as red squares. Error bars in all figures (where they are larger than the symbols) are \pm 1 standard error of the mean (s.e.m.) within observers for the affected individuals, and between observers for the normal measurements.

\section{[Insert Figure 1 about here]}

In normal observers, L-cone CFF starts to rise at about $6.5 \log _{10}$ quanta $\mathrm{s}^{-1} \mathrm{deg}^{-2}$, and above about $7.25 \log _{10}$ quanta s ${ }^{-1} \mathrm{deg}^{-2}$, increases (on these coordinates) with a linear slope until at about 9.75 $\log _{10}$ quanta s $\mathrm{s}^{-1} \mathrm{deg}^{-2}$ it begins to approach a plateau near $40 \mathrm{~Hz} \cdot{ }^{33,34}$ By contrast, the L-cone CFF functions for all four observers with the Tyr99Cys-mutant GCAP1 show substantial losses in CFF. Flicker is not detected in any of the affected observers until the mean 650-nm target radiance reaches at least $7.7 \log _{10}$ quanta $\mathrm{s}^{-1} \mathrm{deg}^{-2}$-nearly 13 times more intense than for normal observers. The differences suggest that the deficit involves a loss of at least $1.2 \log _{10}$ units of intensity. For the affected observers, as the radiance increases above $7.7 \log _{10}$ quanta $\mathrm{s}^{-1} \mathrm{deg}^{-2}$, the CFF increases but, expect for GP3 where no asymptote is apparent, approaches much lower asymptotic CFF values than normal. The CFF loss is greater the older the patient. The highest CFF for the mean normal observer is about $40 \mathrm{~Hz}$ but declines to $29,23,11$, and $10 \mathrm{~Hz}$ for observers GP1 to GP4, respectively (see also Figure 3 and Table 3, below). In terms of temporal acuity, the four observers with the Tyr99Cysmutant GCAP1 show losses that increase with age. 
There is a region both for the normal and for the affected observers over which the CFF is approximately linearly related to the logarithm of the target radiance. This linear relation, known as the Ferry-Porter law, ${ }^{35,36}$ holds, in normal observers, from about 7.25 to $9.75 \log _{10}$ quanta s-1 deg $^{-2}$. For the affected observers, with the exception of GP1, the slope over the Ferry-Porter region is much shallower and the range over which it occurs is displaced to higher radiances. We can quantify and compare the individual CFF data shown in Figure 1 in terms of the slope. The blue straight lines fitted to each set of CFF data shown in Figure 1 are the best-fitting slopes over the Ferry-Porter regions for each observer. The best-fitting slopes, their standard errors and the $R^{2}$ values are given in Table 2 . The slopes for GP2-GP4, which are between 2.91 and $4.42 \mathrm{~Hz}$ per decade, are much less than the normal slope of 8.57 , yet, interestingly, the slope for GP1 at $18.79 \mathrm{~Hz}$ per decade is twice that of the normal slope. The high $R^{2}$ values in Table 2 suggest that the Ferry-Porter law is a plausible description of the data over the appropriate ranges. (The Ferry-Porter slopes are considered further in the Discussion.)

TABLE 2

\begin{tabular}{ccc}
\hline Subject & Ferry-Porter slope & $\boldsymbol{R}^{\mathbf{2}}$ \\
\hline GP1 & $18.79 \pm 0.88$ & 0.996 \\
GP2 & $4.42 \pm 0.27$ & 0.978 \\
GP3 & $3.93 \pm 0.26$ & 0.974 \\
GP4 & $2.91 \pm 0.27$ & 0.960 \\
Normal & $8.57 \pm 0.16$ & 0.998 \\
\hline
\end{tabular}

\section{L-cone temporal contrast sensitivity}

Figure 2 shows the logarithm of temporal modulation sensitivity plotted as a function of temporal frequency (logarithmic axis) for each of the four observers: GP1 (middle left panel, green symbols), GP2 (middle right panel, purple symbols), GP3 (bottom left panel, yellow symbols), GP4 (bottom right panel, blue symbols), and also for the mean normal observer (upper left panel, red symbols). Data are shown for the three time-averaged 650-nm target radiances-Low (8.28, coloured squares), Medium (9.30, coloured triangles), and High (10.28, coloured circles); all radiances in log quanta s $\mathrm{s}^{-1} \mathrm{deg}^{-2}$. The error bars again indicate \pm 1 s.e.m. within observers for the GCAP1 data, across observers for the normal data.

[Insert Figure 2 about here] 
The L-cone temporal contrast sensitivity functions for the mean normal observer (upper left panel) change in two characteristic ways with increasing radiance: ${ }^{29,30,37}$ First, consistent with the CFF measurements shown in Figure 1, the functions extend to higher frequencies as the mean radiance increases from low to high. The improvements in high-frequency sensitivity are usually attributed to a speeding up of the visual response caused by shortening time constants. ${ }^{38-41}$ Second, there is an increasing loss of sensitivity at low frequencies as the radiance increases; the functions change from being relatively horizontal at low frequencies in the low-radiance case (squares) - a shape is known as "low-pass" - to having a sensitivity loss at both low and high frequencies-a shape known as "band-pass" - in the high-radiance case (circles). The bandpass shape is found in both temporal and spatial MTFs and the low-frequency attenuation is usually attributed to surround antagonism ${ }^{42-47}$ but could also result from feedback within the receptors.

The L-cone temporal contrast sensitivities for the observers with Tyr99Cys-mutant GCAP1 fall well below the normal sensitivities at all three mean radiances at all frequencies. (These differences are more clearly apparent in Figure 4 in which the sensitivity losses relative to the normal are plotted.) Despite the losses, some of the changes between levels in the mutant-observers have similar characteristics to the normal. In particular, there is a tendency for high-frequency sensitivity to improve as the radiance level increases, which suggests that, as in normal observers, the photoreceptor response speeds up as the light level increases. Also, for the younger observers GP1 and GP2, there is a tendency to show a low-frequency loss that increases with target radiance. No reliable effect of radiance at low frequencies is seen for the older observers, GP3 and GP4.

Like the CFF data, the overall sensitivity losses increase in order of age from GP1 to GP4. One notable exception is that although GP1 is more sensitive than GP2 at high temporal frequencies (consistent with the CFF data), the reverse is the case at lower frequencies. The sensitivity losses for GP2 relative to normal observers increase from about 0.5 to $1.0 \log _{10}$ unit with increasing frequency. The losses for GP1 relative to normal observers are about $1.0 \log _{10}$ unit at low frequencies but then decrease slightly with increasing frequency. The losses for GP3 and GP4 are between 0.5 and 1.2 $\log _{10}$ unit at low frequencies and increase markedly with increasing frequency.

We consider the relative losses of all the affected observers in more detail after developing a model to account for the losses in the next section. 


\section{DISCUSSION}

\section{L-cone critical flicker fusion}

As noted above, the L-cone CFF functions first plotted in Figure 1 and re-plotted in Figure 3 over a slightly smaller range show that the affected observers require more light to detect the flickering target by at least a factor of 10 times greater than normal. The other characteristic change in the older GCAP1-mutant observers is that their Ferry-Porter slopes are much shallower than normal (see Table 2). Yet, intriguingly, the slope in the youngest GCAP1-mutant observer is steeper than normal. How can we interpret these changes in slopes?

The steepness of the Ferry-Porter slope can be compared with the high-frequency slope of temporal modulation functions plotted as log modulation sensitivity versus linear frequency (rather than against log frequency as shown in Figure 2) simply by rotating the CFF versus log radiance plot clockwise by $90^{\circ} .48,49$ Given that the high-frequency slope of temporal modulation function is related to the speed of the photoreceptor response, ${ }^{28,29,31}$ we might expect a decrease in the FerryPorter slopes for our central $4^{\circ}$ data to correlate with an increase in the high-frequency slope of the contrast sensitivity function, and thus be consistent with a slowing down of the visual response. And, conversely, we might expect an increase in the Ferry-Porter slope to correlate with a decrease in the high-frequency slope of the contrast sensitivity function, and thus with a speeding up of the visual response. Consequently, in the youngest GCAP1-mutant observer in whom the Ferry-Porter slope increases, we would predict a shallower high-frequency modulation sensitivity slope and a speeding up of the response relative to normals, and in the older affected observers, we would predict a steeper high-frequency slope and a slowing down relative to normals. These predictions are borne out in the temporal contrast sensitivity data and the analyses the given in the next section.

TABLE 3

\begin{tabular}{cccc}
\hline Subject & $\begin{array}{c}\text { Relative } \\
\text { shift }\end{array}$ & $\begin{array}{c}\text { Ferry-Porter } \\
\text { slope }\end{array}$ & $\boldsymbol{R}^{\mathbf{2}}$ \\
\hline GP1 & $0.00 \pm 1.85$ & & \\
GP2 & $8.36 \pm 1.88$ & $3.86 \pm 0.20$ & 0.992 \\
GP3 & $15.50 \pm 1.88$ & & \\
GP4 & $18.90 \pm 1.85$ & & \\
\hline
\end{tabular}

The Ferry-Porter slopes for the affected individuals (with the exception of GP1) are relatively similar (see Table 2), so that we can simplify and quantify the progressive loss by assuming that they 
share a common Ferry-Porter slope. (Given the similar CFF slope for GP1 found above a target radiance of $8.5 \log$ quanta $\mathrm{s}^{-1} \mathrm{deg}^{-2}$ (Figure 3), we also included that region of the GP1's CFF data in the analysis.) We can then characterize the increasing losses among the affected observers as vertical shifts in $\mathrm{Hz}$. We estimated the shifts by simultaneously fitting a line of the same slope to the CFF data for all affected observers over their assumed Ferry-Porter ranges (see Figure 3 ). The bestfitting lines with a common slope of $3.86 \mathrm{~Hz}$ per decade are plotted in Figure 3 as blue lines, and the vertical shifts relative to the fit for GP1 are also noted in the figure. The best fitting-parameters and their standard errors are given in Table 3. The $R^{2}$ value, a measure of the goodness of the fit, is 0.992 suggesting that the simplification of having a common slope and accounting for the damage or loss as vertical shifts in CFF is a plausible description of the data. Yet, precisely how the loss in CFF in $\mathrm{Hz}$ can be related to the underlying photoreceptor loss or damage in these observers is less certain. The fact that Ferry-Porter slope is approximately constant suggests that the form of the residual underlying visual response does not change near CFF despite the progressive damage with age. As noted above, a shallower Ferry-Porter slope in $\mathrm{Hz}$ per decade implies a steeper loss in contrast sensitivity at higher temporal frequencies, which is consistent with the damage resulting in a more sluggish visual response. We now address the differences in sensitivity between normal and affected observers.

[Insert Figure 3 about here]

\section{L-cone temporal contrast sensitivity}

Temporal contrast sensitivity data provide more complete information about the visual response than CFF data, and allow us to model the changes caused by the molecular defects. Figure 4 shows better the $\log _{10}$ differences in sensitivity between the mean normal temporal contrast sensitivities and those for the GCAP1-mutant observers: GP1 (green triangles), GP2 (purple circles), GP3 (yellow inverted triangle) and GP4 (blue diamonds) at the high (upper panel), medium (middle panel) and low (lower panel) 650-nm mean radiances. In all panels the $\log _{10}$ difference from the mean normal observer is plotted as a function of frequency (logarithmic scale). (Note that the differences are all negative, which indicates that the GCAP1-mutant observers show sensitivity losses under all the conditions measured.) In each panel the continuous red lines and dashed black lines are model fits and will be discussed below.

\section{[Insert Figure 4 about here]}

Our approach to understanding and modelling the sensitivity differences between the affected and normal data is to assume that the visual process can be treated as cascades of leaky integrating 
stages (or buffered RC circuits), the outputs of which decay exponentially after exposure to a brief pulse of light. The amplitude, $A(f)$, of $n$ cascaded, identical, stages as a function of frequency, $f$, is given by:

$$
A(f)=\tau^{n}\left[(2 \pi f \tau)^{2}+1\right]^{-\frac{n}{2}},
$$

where $\tau$ is the time constant (seconds) common to each stage and $n$ is the number of stages. Light adaptation is assumed to shorten the time constants of some of the stages, and so speeds up the visual response. As Equation 3 shows, the shortening time constant reduces overall sensitivity (by the factor $\tau^{n}$ outside the square bracket) but has the important benefit of allowing more rapid flicker to be seen.

The approach of modelling vision as a linear temporal filter has a long tradition. ${ }^{37,38,47,50,51}$ In terms of phototransduction, the approach can be compared to considering the system as a cascade of independent reactions each having first-order exponential decays. In the leaky integrator, the response to a pulse decays exponentially with time; while in the reaction, the concentration of the reactant decays exponentially with time. We are going to model the differences between normal and affected observers using Equation 3.

By modelling the sensitivity differences between the affected and normal observers, we are effectively discounting receptoral and postreceptoral processes that are common to both classes of observer, and, in particular, any stages of the transduction cascade that have similar time constants. We assume that the differences reflect stages that are common to the two classes of observer but that have different time constants. In fact, we can account for the differences between the Tyr99Cys-mutant GCAP1 and normal observers very simply by assuming that they reflect the properties of a single common stage with a time constant that varies between the normal and affected observers. Put formally, the model is given by Equation [4]:

$$
\log _{10}\left[\frac{A_{N}(f)}{A_{M}(f)}\right]=\log _{10}\left[\frac{\tau_{N}\left[\left(2 \pi f \tau_{N}\right)^{2}+1\right]^{-\frac{1}{2}}}{\tau_{M}\left[\left(2 \pi f \tau_{M}\right)^{2}+1\right]^{-\frac{1}{2}}}\right]+k,
$$

where the subscripts $N$ and $M$ indicate parameters of the normal and mutant frequency responses, respectively. The value of $k$ represents a frequency independent change in overall sensitivity of the GCAP1-mutant observers relative to normals (which as negative values corresponds to a vertical shift down the logarithmic ordinate of Figure 4).

At each level, the model was fitted simultaneously across the four observers with a single bestfitting time constant $\left(\tau_{N}\right)$ for the normal observer, relative to which the best-fitting time constant $\left(\tau_{M}\right)$ and shift $(k)$ could be determined for each affected observer. (The time constants, in general, 
were limited to a lower value of $1.59 \mathrm{~ms}$, because decreasing them below this value, produces frequency-independent shifts in log sensitivity over the measured frequency range that are nearly equivalent to increasing $k$, making joint fits of $\tau$ and $k$ unstable. For the fits in Figure 4 , it was necessary to limit $\tau_{M}$ to 1.59 ms only for GP1.)

The fits to the logarithmic differences, shown by the red continuous lines in each panel of Figure 4 , are good, having $R^{2}$ values of 0.90 or better. The best-fitting parameters and their standard errors are given in Table 4, along with the $R^{2}$ values for the fits at each radiance level. The values in brackets under each time constant are the so-called "corner frequencies", $f_{0}$, associated with each time constant, where $f_{0}=\frac{1}{2 \pi} \tau$ (with $f_{0}$ in $\mathrm{Hz}$ and $\tau$ in seconds). These values are useful in relating the low-pass filters defined by Equation [3] to psychophysical data, because they roughly correspond to the frequency at which the filter first begins to attenuate high-frequencies. Below the corner frequency, sensitivity is fairly constant with frequency.

The corner frequencies associated with the first line of Table 4 suggest that, in the youngest observer (GP1), the excess of cGMP results in the temporal response's being about three times faster (or perhaps more given that we limited $\tau_{M}$ in this observer to $1.59 \mathrm{~ms}$ ) than that of the normal. However with age, and presumably with increasing damage and/or photoreceptor loss, the temporal response from GP2 to GP4 slows down as indicated by the decreasing corner frequencies (and increasing time constants). Across the three levels, the mean time constants for GP1 to GP4 are $1.59,41.43,193.00$ and $132.15 \mathrm{~ms}$, respectively. This increase is consistent with the progressive losses of high-frequency sensitivity found in these observers. The shorter the time constant $\tau$, the faster the system can respond and the more rapid the flicker it can resolve, but the lower its overall sensitivity (depending on $\tau_{M} / \tau_{N}$ in see Equation [4]). The parameter $k$ represents frequencyindependent logarithmic sensitivity losses or gains not accounted for by changes in time constants. The increasing magnitude of $k$ with age is indicative of the decreasing sensitivity of the affected observers with age - a loss that exceeds that due to rising $\tau$.

This simple model accounts extremely well for the relative sensitivity differences between each GCAP1-mutant data and the normal mean. Potentially, the differences can be linked to changes in the reactions mediated by GCAP1. A model in which one stage in the normal retina speeds up in the youngest GCAP1 observer but then becomes more sluggish with age could suggest that as the disease progresses the damaged photoreceptors maintain some functionality but only by means of a sluggish molecular process that limits temporal sensitivity.

It should be noted that if the time constant of the common stage is short in the normal observer but significantly longer in the GCAP1 observers, then the predictions of the model given by Equation 
[4] are indistinguishable in our data from one in which an additional stage is simply added in the GCAP1 observer. Such fits are essentially equivalent to fitting a low-pass filter with a final asymptotic logarithmic slope of -1 to the differences. If, however, the time constants of the common stage in the two classes of observers are more similar, the final measured slope of the differences will decrease towards zero at high frequencies (corresponding to the stage in both observers reaching its final asymptotic logarithmic slope of -1). Many, but not all, of the differences in Figure 4 are consistent with a decrease in the slope at the highest frequencies.

\section{TABLE 4}

\begin{tabular}{|c|c|c|c|c|c|}
\hline & Normal & GP1 & GP2 & GP3 & GP4 \\
\hline \multicolumn{6}{|l|}{ High level } \\
\hline$\tau_{N}$ or $\tau_{M}$ & $\begin{array}{c}5.66 \pm 1.60 \\
(28.11)\end{array}$ & $\begin{array}{l}1.59 * \\
(100)\end{array}$ & $\begin{array}{c}36.05 \pm 7.37 \\
(4.41)\end{array}$ & $\begin{array}{c}123.3 \pm 29.55 \\
(1.29)\end{array}$ & $\begin{array}{c}136.9 \pm 34.64 \\
(1.16)\end{array}$ \\
\hline$k$ & -- & $-0.36 \pm 0.11$ & $-1.31 \pm 0.10$ & $-1.82 \pm 0.11$ & $-2.06 \pm 0.12$ \\
\hline$R^{2}$ & & & 0.94 & & \\
\hline \multicolumn{6}{|c|}{ Medium level } \\
\hline$\tau_{N}$ or $\tau_{M}$ & $\begin{array}{c}19.72 \pm 2.69 \\
(8.07)\end{array}$ & $\begin{array}{l}1.59 * \\
(100)\end{array}$ & $\begin{array}{c}64.59 \pm 13.74 \\
(2.46)\end{array}$ & $\begin{array}{c}310.0 \pm 133.8 \\
(0.53)\end{array}$ & $\begin{array}{c}162.2 \pm 44.55 \\
(0.98)\end{array}$ \\
\hline$K$ & -- & $0.10 \pm 0.04$ & $-1.05 \pm 0.04$ & $-1.64 \pm 0.05$ & $-1.79 \pm 0.05$ \\
\hline$R^{2}$ & & & 0.96 & & \\
\hline \multicolumn{6}{|l|}{ Low level } \\
\hline$\tau_{N}$ or $\tau_{M}$ & $\begin{array}{c}19.58 \pm 5.64 \\
(8.13)\end{array}$ & $\begin{array}{l}1.59 * \\
(100)\end{array}$ & $\begin{array}{c}23.65 \pm 9.14 \\
(6.73)\end{array}$ & $\begin{array}{c}146.6 \pm 69.10 \\
(1.09)\end{array}$ & $\begin{array}{c}97.36 \pm 37.59 \\
(1.63)\end{array}$ \\
\hline$K$ & -- & $-0.07 \pm 0.09$ & $-0.75 \pm 0.08$ & $-1.97 \pm 0.15$ & $-1.77 \pm 0.13$ \\
\hline$R^{2}$ & & & 0.90 & & \\
\hline
\end{tabular}

Although the single-stage model defined by Equation [4] has the virtue of simplicity, a physiologically more plausible model might be a two-stage one in which a first common stage speeds up in all affected observers (due to the direct effect of the mutant proteins) but then a second common stage slows down as the disease progresses. This version of the model is formalized in Equation [5]:

$$
\log _{10}\left[\frac{A_{N}(f)}{A_{M}(f)}\right]=\log _{10}\left[\frac{\tau_{N 1}\left[\left(2 \pi f \tau_{N 1}\right)^{2}+1\right]^{-\frac{1}{2}} \tau_{N 2}\left[\left(2 \pi f \tau_{N 2}\right)^{2}+1\right]^{-\frac{1}{2}}}{\tau_{M 1}\left[\left(2 \pi f \tau_{M 1}\right)^{2}+1\right]^{-\frac{1}{2}} \tau_{M 2}\left[\left(2 \pi f \tau_{M 2}\right)^{2}+1\right]^{-\frac{1}{2}}}\right]+k
$$

The best-fitting parameters and their standard errors of this model are given in Table 5, along 
with the $R^{2}$ values for the fits at each radiance level. We constrained the fit by fixing the time constant of the first common stage in the GCAP1-mutant observers $\left(\tau_{M 1}\right)$ to $1.59 \mathrm{~ms}$. The fits are shown as the black dashed lines in each panel of Figure 4 . They are marginally better than those for the common stage at the low and perhaps medium levels. For these fits, other than the constraint noted above, it was necessary only to limit $\tau_{M 2}$ for GP1 to $1.59 \mathrm{~ms}$.

\section{TABLE 5}

\begin{tabular}{|c|c|c|c|c|c|}
\hline & Normal & GP1 & GP2 & GP3 & GP4 \\
\hline \multicolumn{6}{|l|}{ High level } \\
\hline$\tau_{N 1}$ or $\tau_{M 1}$ & $\begin{array}{c}4.35 \pm 1.52 \\
(36.59)\end{array}$ & \multicolumn{4}{|c|}{$\begin{array}{l}1.59 * \\
(100)\end{array}$} \\
\hline$\tau_{\mathrm{N} 2}$ or $\tau_{\mathrm{M} 2}$ & $\begin{array}{c}4.35 \pm 1.52 \\
(36.59)\end{array}$ & $\begin{array}{c}2.94 \pm 4.33 \\
(100)\end{array}$ & $\begin{array}{c}37.21 \pm 8.90 \\
(4.41)\end{array}$ & $\begin{array}{c}126.0 \pm 32.10 \\
(1.29)\end{array}$ & $\begin{array}{c}137.9 \pm 35.77 \\
(1.16)\end{array}$ \\
\hline$k$ & -- & $-0.30 \pm 0.41$ & $-1.00 \pm 0.25$ & $-1.50 \pm 0.28$ & $-1.74 \pm 0.29$ \\
\hline$R^{2}$ & \multicolumn{5}{|c|}{0.94} \\
\hline \multicolumn{6}{|c|}{ Medium level } \\
\hline$\tau_{N 1}$ or $\tau_{M 1}$ & $\begin{array}{c}9.67 \pm 0.91 \\
(16.46)\end{array}$ & \multicolumn{4}{|c|}{$\begin{array}{l}1.59^{*} \\
(100)\end{array}$} \\
\hline$\tau_{N 2}$ or $\tau_{M 2}$ & $\begin{array}{c}9.67 \pm 0.91 \\
(16.46\end{array}$ & $\begin{array}{l}1.59^{*} \\
(100)\end{array}$ & $\begin{array}{c}56.91 \pm 11.70 \\
(2.80)\end{array}$ & $\begin{array}{c}235.1 \pm 79.54 \\
(0.68)\end{array}$ & $\begin{array}{c}139.7 \pm 35.97 \\
(1.14)\end{array}$ \\
\hline$\kappa$ & -- & $0.61 \pm 0.06$ & $-0.52 \pm 0.07$ & $-1.12 \pm 0.08$ & $-1.27 \pm 0.08$ \\
\hline$R^{2}$ & & & 0.96 & & \\
\hline
\end{tabular}

Low level

\begin{tabular}{cc|cccc} 
& & \multicolumn{4}{|c}{$1.59 *$} \\
$\tau_{N 1}$ or $\tau_{M 1}$ & $20.08 \pm 5.33$ & $(7.93)$ & \multicolumn{4}{|c}{$(100)$} \\
& $20.08 \pm 5.33$ & $22.08 \pm 12.64$ & $60.33 \pm 28.76$ & $171.1 \pm 77.40$ & $120.1 \pm 47.19$ \\
$\tau_{N 2}$ or $\tau_{M 2}$ & $(7.93)$ & $(7.21)$ & $(2.64)$ & $(0.93)$ & $(1.33)$ \\
$K$ & - & $-0.09 \pm 0.07$ & $0.03 \pm 0.09$ & $-0.91 \pm 0.21$ & $-0.73 \pm 0.18$ \\
$K$ & & & 0.92 & &
\end{tabular}

From the point of view of the quality of the fit, there is no clear advantage in adding a second common stage. However, the addition of this second stage lends itself to very different interpretations at the molecular level, since although the progressively slowing stage could be in the photoreceptor, it could also be after the photoreceptor. The slowing, for example, could result from the way signals are processed in the postreceptoral network as photoreceptor inputs become increasingly scarce. For example, with increasing loss the predominant and most effective cone signal might come from a spatially-extensive and sluggish surround rather than from the centre as in 
normal observers.

Alternatively, the photoreceptor loss might result in an active rewiring and reorganization ${ }^{52,53}$ that produces a novel postreceptoral organization not found in the normal retina.

\section{In vivo murine models}

The psychophysical results obtained in the youngest individual suggest that the Tyr99Cysmutation speeds up the cone visual response. However, this finding is at odds with the murine rod data described in the Introduction, which-at least in terms of $\tau_{\mathrm{D}}$, (or $\tau_{\text {sat }}$ ), the dominant time constant of exponential recovery after saturation-suggest that the $\mathrm{Ca}^{2+}$-dependent resynthesis of cGMP is not rate-limiting. In addition, other work in which the expression level or activity of molecules in the cascade was perturbed also supports the idea that the rate limiting recovery step is the deactivation of $\alpha$-transducin-PDE6* and not cGMP resynthesis. ${ }^{25,54,55}$

However, the usefulness of the two key measures of rod flash responses, $\tau_{\text {rec }}$ and $\tau_{D}$, in predicting cone visual performance measured under conditions of steady-state (equilibrium) daylight adaptation using non-saturating, near-threshold stimuli (as in our experiments) may be limited. The inconsistences between the murine and human data could be due to differences between rods and cones, or they could be due to the dynamics that control recovery from saturation being different from those that limit the detection of near-threshold flicker under conditions of steady-state adaptation.

One set of data suggest that there might be significant differences between the effects of GCAP1 on rod and cone responses. Pennesi et al. ${ }^{56}$ measured paired-flash cone ERGs in GCAP1/GCAP2 knockout mice that expressed transgenic GCAP1. They found that overexpression of GCAP1 resulted in the cone b-wave recovering faster than in the normal, wild-type mouse. Thus, in this murine model at least GCAP1 can alter the speed of the cone visual response, which suggests that in cones the resynthesis of cGMP might be rate limiting.

Alternatively, the speeding up of the visual response in the youngest observer could be an early sign of photoreceptor degeneration. One way to check this would be to be make measurements in even younger observers, but we were unable to make measurements in the younger generation of this family. Age comparisons is an in vivo murine Tyr99Cys-cone model would be informative.

Elevated levels of cGMP in photoreceptors caused by the Tyr99Cys-mutation result in photoreceptor degeneration in mice. ${ }^{23}$ Recent evidence implicates cGMP accumulation as the major contributor to cone death caused by cyclic-nucleotide gated CNG channel deficiency. ${ }^{57}$ 


\section{CONCLUSIONS}

Evidence for a shortening of the integration time at lower light levels in the Tyr99Cys-mutant GCAP1 observers was found only in the youngest of the observers, who was 39 years of age. In all other observers there was a substantial higher-frequency sensitivity loss-presumably caused by the progressive damage-that masks any speeding-up due to the GCAP1 mutation. We suppose that these additional losses are due to either a limiting sluggish molecular process that maintains some function within damaged photoreceptors, or to a postreceptoral effect related to the sparseness of photoreceptors.

\section{ACKNOWLEDGEMENTS}

This work was supported by grants from Fight for Sight, BBSRC, EPSRC, Moorfields Eye Hospital Special Trustees, and the National Institute for Health Research Biomedical Research Centre at Moorfields Eye Hospital NHS Foundation Trust and UCL Institute of Ophthalmology. ARW, ATM and MM are supported by a Foundation Fighting Blindness Research Center grant for the Study of Retinal Degenerative Diseases. MM is supported by a Foundation Fighting Blindness Career Development Award. We especially acknowledge the help of the observers who participated in this study without whom this work would not have been possible.

\section{REFERENCES}

1. Perlman I, Normann RA. Light adaptation and sensitivity controlling mechanisms in vertebrate photoreceptors. Progress in Retinal and Eye Research 1998;17:523-563.

2. Pugh EN, Jr., Nikonov S, Lamb TD. Molecular mechanisms of vertebrate photoreceptor light adaptation. Current Opinion in Neurobiology 1999;9:410-418.

3. Pugh EN, Jr., Lamb TD. Phototransduction in vertebrate rods and cones: molecular mechanisms of amplification, recovery and light adaptation. In: Stavenga DG, de Grip WJ, Pugh EN (eds), Handbook of biological physics, Vol 3, Molecular mechanisms of visual transduction. Amsterdam: Elsevier; 2000:183-255.

4. Burns ME, Baylor DA. Activation, deactivation and adaptation in vertebrate photoreceptor cells. Annual Review of Neuroscience 2001;24:779-805.

5. Arshavsky VY, Lamb TD, Pugh EN, Jr. G proteins and phototransduction. Annual review of physiology 2002;64:153-187. 
6. Fain GL, Matthews HR, Cornwall MC, Koutalos Y. Adaptation in vertebrate photoreceptors. Physiological Reviews 2001;80:117-151.

7. Burns ME, Mendez A, Chen J, Baylor DA. Dynamics of cyclic GMP synthesis in retinal rods. Neuron 2002;36:81-91.

8. Liu X, Seno K, Nishizawa Y, et al. Ultrastructural localization of retinal guanylate cyclase in human and monkey retinas. Experimental eye research 1994;59:761-768.

9. Yang RB, Foster DC, Garbers DL, Fulle HJ. Two membrane forms of guanylyl cyclase found in the eye. Proceedings of the National Academy of Science USA 1995;92:602-606.

10. Dizhoor AM, Olshevskaya EV, Henzel WJ, et al. Cloning, sequencing, and expression of a 24-kDa $\mathrm{Ca}(2+)$-binding protein activating photoreceptor guanylyl cyclase. Journal of Biological Chemistry 1995;270:25200-25206.

11. Gorczyca WA, Polans AS, Surgucheva IG, Subbaraya I, Baehr W, Palczewski K. Guanylyl cyclase activating protein. A calcium-sensitive regulator of phototransduction. The Journal of biological chemistry 1995;270:22029-22036.

12. Imanishi Y, Li N, Sokal I, et al. Characterization of retinal guanylate cyclase-activating protein 3 (GCAP3) from zebrafish to man. European Journal of Neuroscience 2002;15:63-78.

13. Payne AM, Downes SM, Bessant DA, et al. A mutation in guanylate cyclase activator $1 A$ (GUCA1A) in an autosomal dominant cone dystrophy pedigree mapping to a new locus on chromosome 6p21.1. Human Molecular Genetics 1998;7:273-277.

14. Cuenca N, Lopez S, Howes K, Kolb H. The localization of guanylyl cyclase-activating proteins in the mammalian retina. Investigative ophthalmology \& visual science 1998;39:1243-1250.

15. Dizhoor AM, Lowe DG, Olshevskaya EV, Laura RP, Hurley JB. The human photoreceptor membrane guanylyl cyclase, RetGC, is present in outer segments and is regulated by calcium and a soluble activator. Neuron 1994;12:1345-1352.

16. Ermilov AN, Olshevskaya EV, Dizhoor AM. Instead of binding calcium, one of the EF-hand structures in guanylyl cyclase activating protein-2 is required for targeting photoreceptor guanylyl cyclase. The Journal of biological chemistry 2001;276:48143-48148.

17. Hunt DM, Buch P, Michaelides M. Guanylate cyclases and associated activator proteins in retinal disease. Molecular and Cellular Biochemistry 2010;334:157-168.

18. Dizhoor AM, Boikov SG, Olshevskaya EV. Constitutive activation of photoreceptor guanylate cyclase by Y99C mutant of GCAP-1. Possible role in causing human autosomal dominant cone degeneration. The Journal of biological chemistry 1998;273:17311-17314.

19. Sokal I, Li N, Surgucheva I, et al. GCAP1 (Y99C) mutant is constitutively active in autosomal dominant cone dystrophy. Molecular Cell 1998;2:129-133. 
20. Downes SM, Holder GE, Fitzke FW, et al. Autosomal dominant cone and cone-rod dystrophy with mutations in the guanylate cyclase activator $1 \mathrm{~A}$ gene-encoding guanylate cyclase activating protein1. Archives of Ophthalmology 2001;119:96-105.

21. Michaelides M, Wilkie SE, Jenkins S, et al. Mutation in the gene GUCA1A, encoding guanylate cyclase-activating protein 1, causes cone, cone-rod, and macular dystrophy. Ophthalmology 2005;112:1442-1447.

22. Michaelides M, Hardcastle AJ, Hunt DM, Moore AT. Progressive cone and cone-rod dystrophies: phenotypes and underlying molecular genetic basis. Survey of Ophthalmology 2006;51:232-258.

23. Olshevskaya EV, Calvert PD, Woodruff ML, et al. The Y99C mutation in guanylyl cyclase-activating protein 1 increases intracellular $\mathrm{Ca} 2+$ and causes photoreceptor degeneration in transgenic mice. The Journal of Neuroscience 2004;24:6078-6085.

24. Mendez A, Burns ME, Sokal I, et al. Role of guanylate cyclase-activating proteins (GCAPs) in setting the flash sensitivity of rod photoreceptors. roceedings of the National Academy of Sciences of the United States of America 2001;98:9948-9953.

25. Burns ME. Deactivation Mechanisms of Rod Phototransduction The Cogan Lecture. Investigative ophthalmology \& visual science 2010;51:1283-1288.

26. Lyubarsky A, Nikonov S, Pugh EN, Jr. The kinetics of inactivation of the rod phototransduction cascade with constant Ca2+i. The Journal of general physiology 1996;107:19-34.

27. Nikonov S, Engheta N, Pugh EN, Jr. Kinetics of recovery of the dark-adapted salamander rod photoresponse. Journal of General Physiology 1998;111:7-37.

28. Stockman A, Langendörfer M, Smithson HE, Sharpe LT. Human cone light adaptation: from behavioral measurements to molecular mechanisms. Journal of Vision 2006;6:1194-1213.

29. de Lange $\mathrm{H}$. Research into the dynamic nature of the human fovea-cortex systems with intermittent and modulated light. I. Attenuation characteristics with white and colored light. Journal of the Optical Society of America 1958;48:777-784.

30. Kelly DH. Visual responses to time-dependent stimuli I. Amplitude sensitivity measurements. Journal of the Optical Society of America 1961;51:422-429.

31. Roufs JAJ. Dynamic properties of vision-I. Experimental relationships between flicker and flash thresholds. Vision Research 1972;12:261-278.

32. Stockman A, Plummer DJ, Montag ED. Spectrally-opponent inputs to the human luminance pathway: slow $+M$ and $-L$ cone inputs revealed by intense long-wavelength adaptation. Journal of Physiology 2005;566:61-76. 
33. Hecht $S$, Verrijp CD. The influence of intensity, color and retinal location on the fusion frequency of intermittent illumination. Proceedings of the National Academy of Sciences of the United States of America 1933;19:522-535.

34. Hecht S, Shlaer S. Intermittent stimulation by light. V. The relation between intensity and critical frequency for different parts of the spectrum. Journal of General Physiology 1936;19:965-977.

35. Ferry J. Persistence of vision. American Journal of Science and Arts 1892;44:192-207.

36. Porter TC. Contributions to the study of flicker. II. Proceedings of the Royal Society of London Series A 1902;70:313-319.

37. Roufs JAJ. Dynamic properties of vision. II. Theoretical relationship between flicker and flash thresholds. Vision Research 1972;12:279-292.

38. Ives HE. A theory of intermittent vision. Journal of the Optical Society of America 1922;6:343361.

39. Fourtes MGF, Hodgkin AL. Changes in time scale and sensitivity in the ommatidia of Limulus. Journal of Physiology 1964;172:239-263.

40. Matin L. Critical duration, the differential luminance threshold, critical flicker frequency, and visual adaptation: a theoretical treatment. Journal of the Optical Society of America 1968;58:404415.

41. Sperling G, Sondhi MM. Model for visual luminance discrimination and flicker detection. Journal of the Optical Society of America 1968;58:1133-1145.

42. Schober HAW, Hilz R. Contrast sensitivity of the human eye for square-wave gratings. Journal of the Optical Society of America 1965;55:1086-1091.

43. Robson JG. Spatial and temporal contrast sensitivity functions of the visual system. Journal of the Optical Society of America 1966;56:1141-1142.

44. Nachmias J. Effect of exposure duration on visual contrast sensitivity with square-wave gratings. Journal of the Optical Society of America 1967;57:421-427.

45. Ratliff F, Knight BW, Toyoda J-I, Hartline HK. Enhancement of flicker by lateral inhibition. Science 1967;158:392-393.

46. Kelly DH. Flickering patterms and lateral inhibition. Journal of the Optical Society of America 1969;59:1361-1370.

47. Watson AB. Temporal sensitivity. In: Boff K, Kaufman L, Thomas J (eds), Handbook of Perception and Human Performance. New York: Wiley; 1986:6-1-6-43.

48. Tyler CW. Analysis of visual modulation sensitivity. II. Peripheral retina and the role of photoreceptor dimensions. Journal of the Optical Society of America A 1985;2:393-398. 
49. Tyler CW, Hamer RD. Analysis of visual modulation sensitivity. IV. Validity of the Ferry-Porter law. Journal of the Optical Society of America A 1990;7:743-758.

50. de Lange $\mathrm{H}$. Experiments on flicker and some calculations on an electrical analogue of the foveal systems. Physica 1952;18:935-950.

51. Kelly DH. Visual responses to time-dependent stimuli II. Single-channel model of the photopic visual system. Journal of the Optical Society of America 1961;51:747-754.

52. Jones $\mathrm{BW}$, Watt $\mathrm{CB}$, Frederick JM, et al. Retinal remodeling triggered by photoreceptor degenerations. Journal of Comparative Neurology 2003;464:1-16.

53. Marc RE, Jones BW, Watt CB, Strettoi E. Neural remodeling in retinal degeneration. Progress in Retinal and Eye Research 2003;22:607-655.

54. Burns ME, Pugh EN, Jr. RGS9 concentration matters in rod phototransduction. Biophysics Journal 2009;97:1538-1547.

55. Krispel CM, Chen D, Melling N, et al. RGS expression rate-limits recovery of rod photoresponses. Neuron 2006;51:409-416.

56. Pennesi ME, Howes KA, Baehr W, Wu SM. Guanylate cyclase-activating protein (GCAP) 1 rescues cone recovery kinetics in GCAP1/GCAP2 knockout mice. Proceedings of the National Academy of Sciences of the United States of America 2003;100:6783-6788.

57. Xu J, Morris L, Thapa A, et al. cGMP accumulation causes photoreceptor degeneration in CNG channel deficiency: evidence of cGMP cytotoxicity independently of enhanced CNG channel function. Journal of Neuroscience 2013;33:14939-14948.

\section{FIGURE LEGENDS}

Figure 1. L-cone critical flicker fusion frequencies $(\mathrm{Hz}$, linear scale) measured on a $481-\mathrm{nm}$ background of $8.26 \log _{10}$ quanta s$~^{-1} \mathrm{deg}^{-2}$ are plotted as a function of the mean log radiance of a 650 $\mathrm{nm}$ flickering target. Data are plotted for four observers with the Tyr99Cys-mutant GCAP1: GP1 (green triangles), GP2 (purple circles), GP3 (yellow inverted triangles) and GP4 (blue diamonds). The mean data for 12 normal observers (red squares) are also shown. In all figures, the error bars are \pm 1 standard error of the mean (s.e.m.) for the affected observers, and between observers for the mean data. The blue lines are best-fitting linear slopes fitted to the mean data for normals and affected observer over radiances where the Ferry-Porter law holds (see text for details). Outside the FerryPorter regions the CFF data are joined by dashed lines. The best-fitting slopes are given in Table 1. 
Figure 2. $\log _{10} \mathrm{~L}$-cone temporal contrast sensitivities, measured using a sinusoidally modulated 650 $\mathrm{nm}$ target fixed at mean radiances of either 8.28 (squares), 9.30 (triangles), or 10.28 (circles) log quanta $\mathrm{s}^{-1} \mathrm{deg}^{-2}$ are plotted as a function of temporal frequency (logarithmic axis). Each target was superimposed on a $480-\mathrm{nm}$ background of $8.29 \log _{10}$ quanta s $\mathrm{s}^{-1} \mathrm{deg}^{-2}\left(1.42 \log _{10}\right.$ photopic trolands or $2.58 \log _{10}$ scotopic trolands). Data are shown for the four observers with Tyr99Cys-mutant GCAP1: GP1 (middle left panel, green symbols), GP2 (middle right panel, purple symbols), GP3 (bottom left panel, yellow symbols), GP4 (bottom right panel, blue symbols), and also for the mean normal observer (upper right panel, red symbols).

Figure 3. L-cone critical flicker fusion frequencies ( $\mathrm{Hz}$, linear scale) for the Tyr99Cys-mutant GCAP1 observers replotted using the same symbols as in Figure 1 . The blue lines fitted to the affected CFF data at radiances where the Ferry-Porter law holds (see text for details) are lines with a common Ferry-Porter slope shifted vertically to fit the CFF data for each observer. The common best-fitting slope is $3.86 \mathrm{~Hz}$ per decade, and the best-fitting vertical shifts relative to the fit for GP1 are noted in the figure. Outside the Ferry-Porter regions the CFF data are joined by dashed lines. See Table 2 for more information.

Figure 4. $\log _{10} \mathrm{~L}$-cone temporal contrast sensitivity differences from Figure 2 between the mean normal observer and the Tyr99Cys-mutant GCAP1 observers: GP1 (green triangles), GP2 (purple circles), GP3 (yellow inverted triangles) and GP4 (blue diamonds) for mean 650-nm target radiances of 8.28 (lower panel), 9.30 (middle panel), and 10.28 (upper panel) log quanta s $\mathrm{s}^{-1} \mathrm{deg}^{-2}$. The continuous red lines and dashed black lines are fits of the two models described in the text.

\section{TABLE LEGENDS}

Table 1. The gender, age at testing, genotype and visual acuities in the tested eye for observers GP1 to GP4.

Table 2. The best-fitting Ferry-Porter slopes and standard errors in $\mathrm{Hz}$ per decade and $R^{2}$ values for fits to the CFF versus log radiance data for each of the affected observers and for the mean normal observer data at radiances where the Ferry-Porter law holds (see Figure 1). 
Table 3. The best-fitting vertical shifts and standard errors in $\mathrm{Hz}$, the common Ferry-Porter slope and standard error in $\mathrm{Hz}$ per decade, and the $R^{2}$ value for the fit to the CFF versus log radiance data for the affected observers at radiances where the Ferry-Porter law holds.

Table 4. The best-fitting parameters and their standard errors and $R^{2}$ values of the model given by Equation [4] for the High, Medium and Low adaptation levels. See text for details.

Table 5. The best-fitting parameters and their standard errors and $R^{2}$ values of the model given by Equation [5] for the High, Medium and Low adaptation levels. See text for details. 


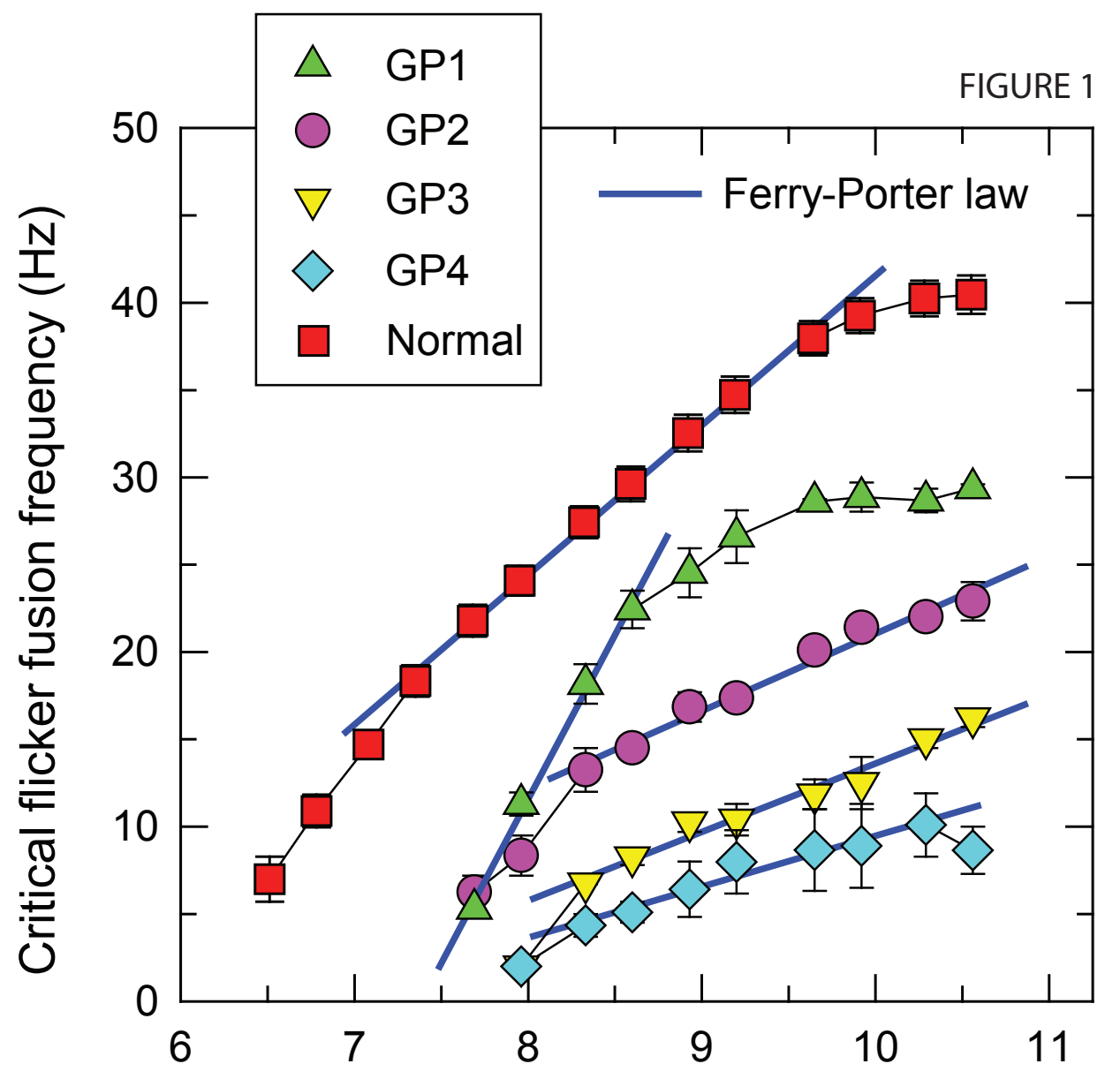

Time-averaged target radiance $\left(\log _{10}\right.$ quanta s $\left.{ }^{-1} \mathrm{deg}^{-2}\right)$ 
FIGURE 2

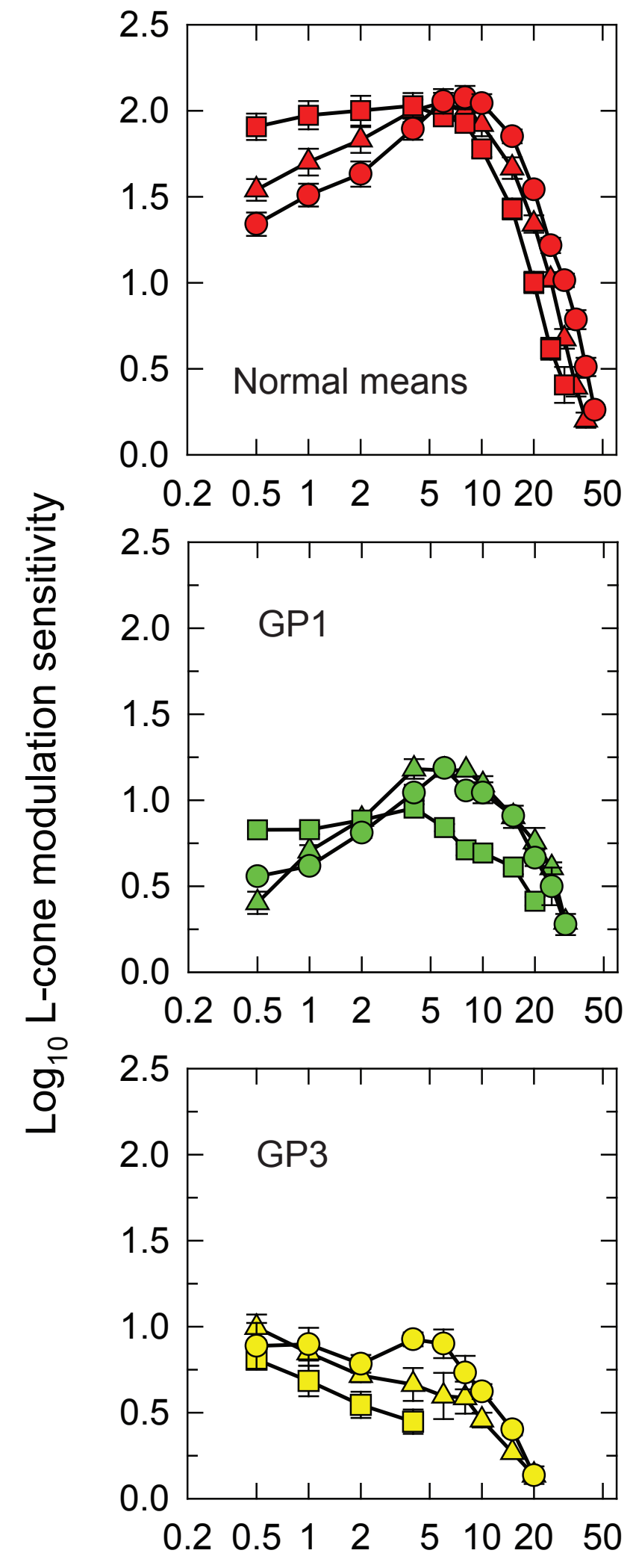

\begin{tabular}{|cl|}
\hline \multicolumn{2}{|c|}{ Levels } \\
$\square$ & Low \\
$\triangle$ & Medium \\
$\bigcirc$ & High \\
\hline
\end{tabular}
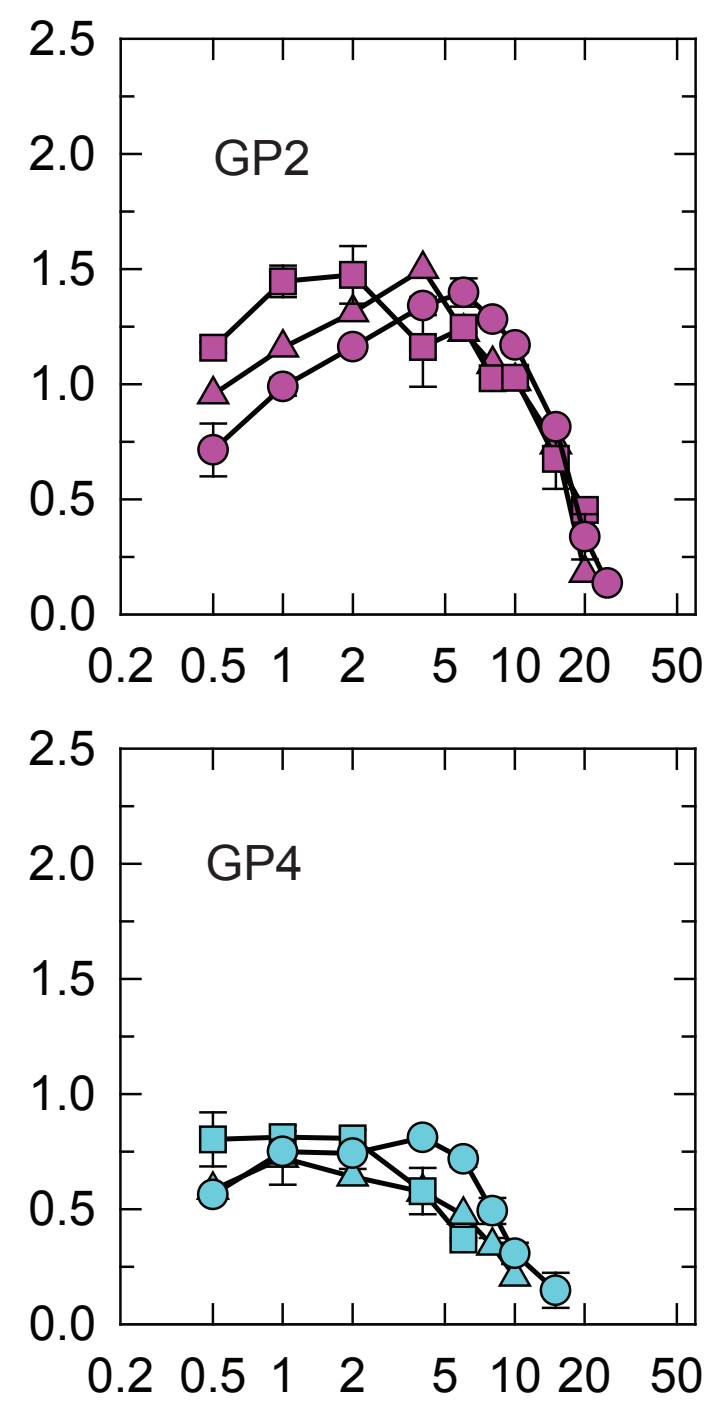

Frequency $(\mathrm{Hz})$ 


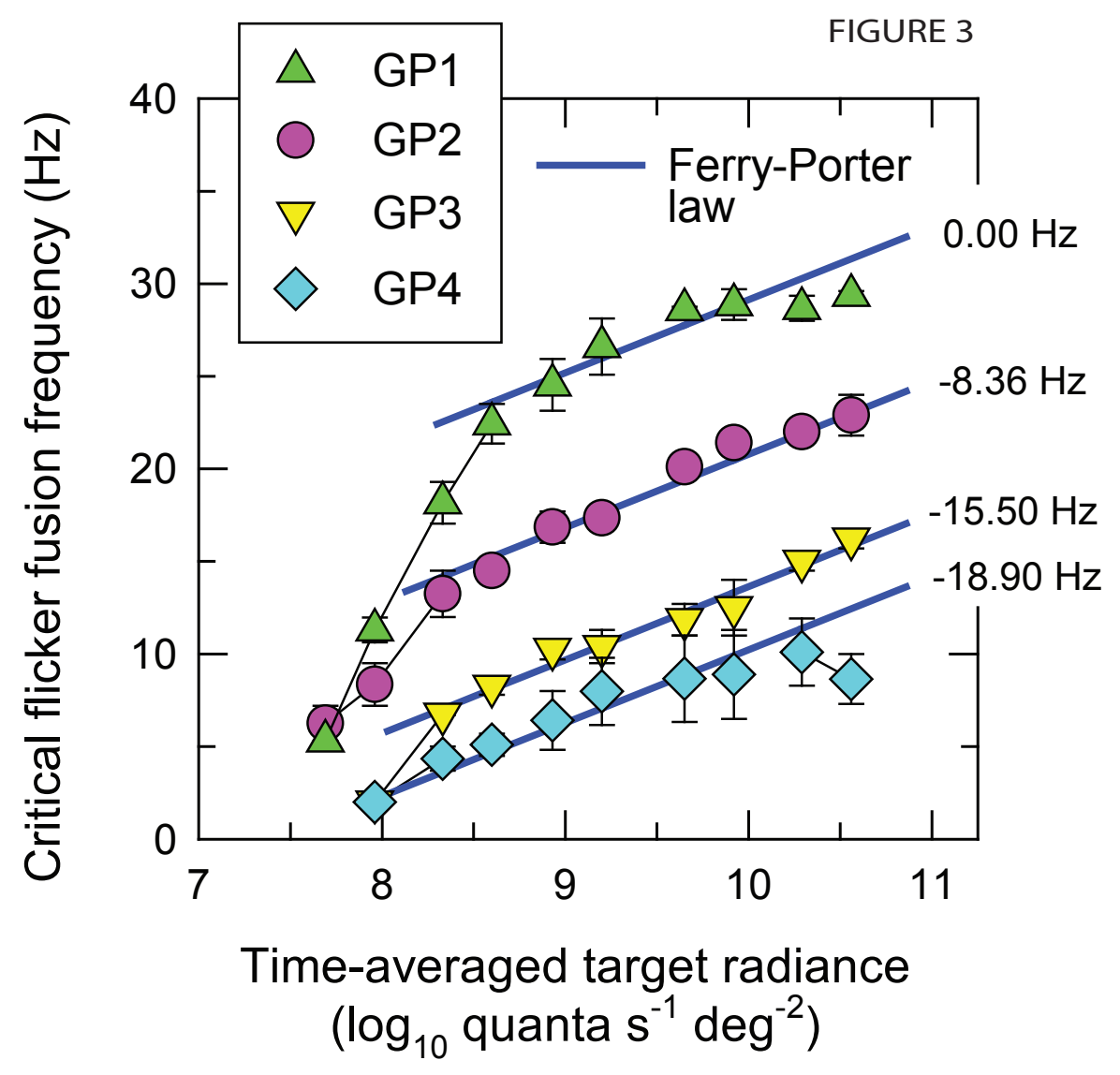




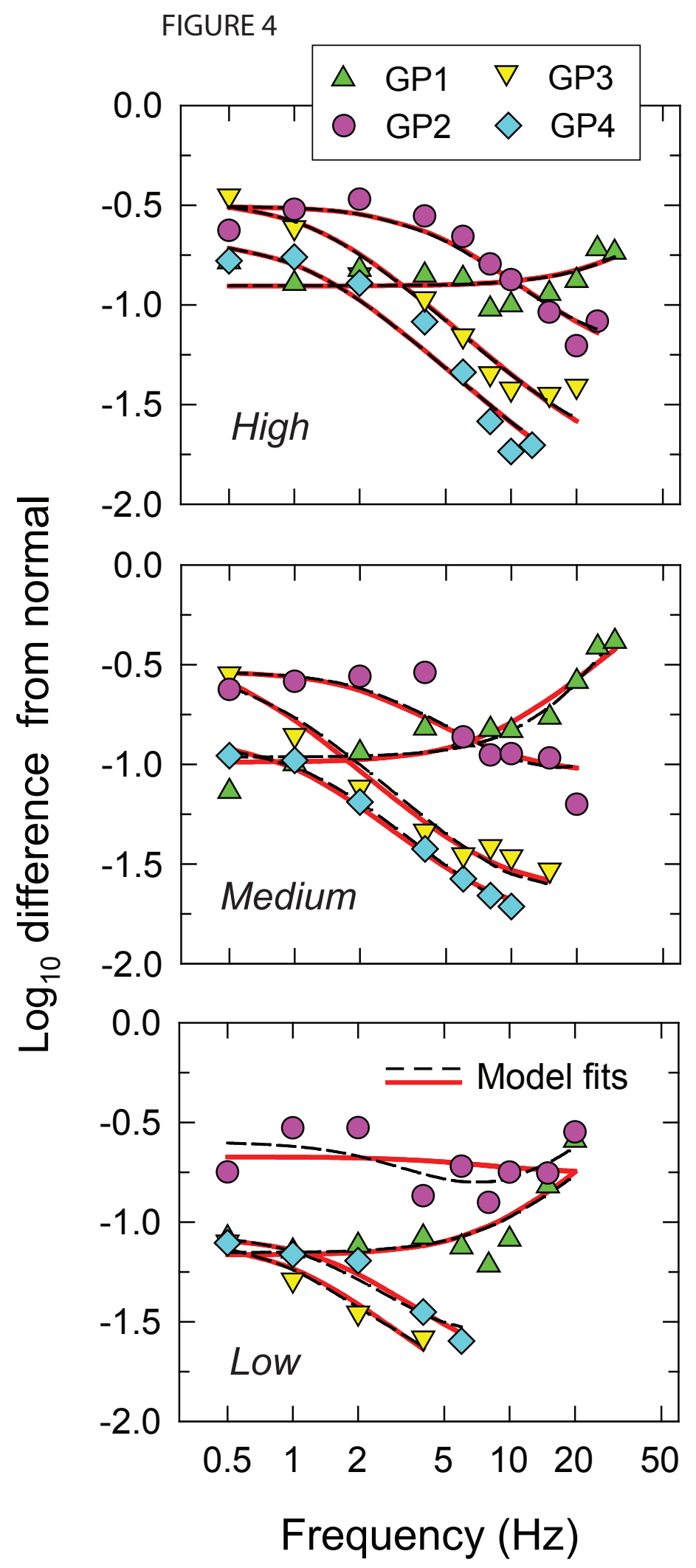

